\title{
La privatisation de la politique étrangère en Asie du Sud
}

« Modes populaires d'action diplomatique » et pratiques de sous-

traitance stratégique en Inde et au Pakistan

\section{Laurent Gayer}

\section{(2) OpenEdition}

\section{Journals}

Édition électronique

URL : http://journals.openedition.org/transcontinentales/559

ISBN : 978-2-8218-1112-6

ISSN : $1775-397 X$

Éditeur

Editions de la maison des sciences de l'homme

\section{Édition imprimée}

Date de publication : 31 décembre 2006

Pagination : 103-127

ISBN : 978-2-200-92170-5

ISSN : 1950-1684

Référence électronique

Laurent Gayer, «La privatisation de la politique étrangère en Asie du Sud », Transcontinentales [En ligne], 3 | 2006, document 7, mis en ligne le 12 octobre 2011, consulté le 07 septembre 2020. URL: http://journals.openedition.org/transcontinentales/559

Ce document a été généré automatiquement le 7 septembre 2020

Tous droits réservés 


\section{La privatisation de la politique étrangère en Asie du Sud}

« Modes populaires d'action diplomatique » et pratiques de soustraitance stratégique en Inde et au Pakistan

Laurent Gayer

1 Dans la première moitié du XVII siècle, la raison d'État s'est imposée en Europe comme la nouvelle "matrice de rationalité » guidant les actions du Prince à l'intérieur mais aussi à l'extérieur des frontières ${ }^{1}$. Dans ces États émergents, qui contribuèrent au cours des siècles suivants à la diffusion transnationale du modèle de l'État-nation, la conduite de la diplomatie et son corollaire, la préparation de la guerre, se sont progressivement imposées comme une prérogative régalienne du pouvoir souverain, à la suite $d u$ " "désarmement" des activités transnationales non-étatiques ${ }^{2}$ ». Les États postcoloniaux ont tous repris à leur compte cette norme, pour dissuader leurs citoyens de conduire leur propre politique étrangère. Ainsi le Logan Act de 1799 interdit-il aux citoyens américains de rencontrer des dirigeants étrangers sans autorisation préalable du Département d'État. Et bien qu'un seul individu ait jusqu'à présent été traduit en justice en vertu de cette loi, et qu'il ait finalement été acquitté, ce texte a servi d'outil dissuasif aux diplomates américains, notamment au cours de la guerre du Vietnam ${ }^{3}$.

2 Toutes les tentatives d'instauration d'un monopole d'État sur la représentation internationale des individus sont cependant restées vaines ${ }^{4}$. La culture, les liens de parenté, les ententes économiques et les convictions religieuses et idéologiques fournissent aux individus des registres de mobilisation qui transcendent le cadre étroit du territoire et qui constituent autant de modes de projection dans la société mondiale. Ces "modes populaires d'action diplomatique" s'enracinent dans des pratiques transnationales anciennes. On rappellera ici la contribution des grandes compagnies à l'entreprise coloniale, mais aussi la fertilité du "long XIX* siècle ", véritable matrice de notre globalisation « post-coloniale ${ }^{5}$, en matière de mouvements transnationaux ${ }^{6}$. Au cours des années 1930 , la dérive antisémite du régime nazi donna pour sa part naissance au premier mouvement transnational de diplomatie identitaire ${ }^{7}$. Au cours de la guerre froide, enfin, trop souvent interprétée comme un banal affrontement de 
puissances étatiques, les initiatives diplomatiques privées se sont multipliées, comme l'illustrent les actions entreprises dans le domaine de la diplomatie culturelle par les organisations de jeunesse, par la communauté scientifique et par les grandes fondations américaines vis-à-vis de l'Union soviétique ${ }^{8}$, ainsi que les entreprises de diplomatie identitaire conduites par les populations indigènes ${ }^{9}$ et par les groupes nationalistes en lutte pour leur autonomie ou leur indépendance ${ }^{10}$.

3 L'histoire longue de ces mobilisations transnationales témoigne de la capacité persistante des acteurs privés à configurer leur environnement international. À travers leurs diplomaties privées, ces acteurs peuvent chercher à seconder leur État ou certains de ses dirigeants. Dans une veine plus subversive de l'ordre interétatique mondial, les "diplomates officieux ${ }^{11}$ " peuvent aussi trouver dans ces projections internationales un moyen d'échapper aux astreintes des régimes juridiques nationaux et une voie d'accès à la reconnaissance internationale, servant à légitimer leur personne, leur organisation ou leur propre projet étatique.

4 Si ces «modes populaires d'action diplomatique» témoignent des capacités persistantes des individus et des groupes sociaux à produire leur propre politique étrangère, dans les interstices de la souveraineté, la privatisation de la politique étrangère est irréductible à ces diplomaties privées. Comme le souligne François Constantin, cette notion recouvre deux types de processus internationaux: "[les] situations où des acteurs privés sont à l'origine de processus induisant des comportements internationaux auxquels les gouvernements doivent réagir », mais aussi « [les] situations où les gouvernements eux-mêmes s'appuient sur des relations de nature privée pour mener soit une politique publique [...] soit une politique personnelle $»^{12}$.

5 Cette distinction a d'importantes implications théoriques. Elle relativise en effet l'hypothèse d'une déficience de la souveraineté dans le champ de la diplomatie, que semble à première vue accréditer la banalité des mobilisations transnationales. Certes, les politiques étrangères officielles peuvent se trouver perturbées par les mobilisations de certains diplomates officieux, mais les agents de l'État peuvent aussi déléguer à leurs partenaires sociaux des missions internationales. Lorsque celles-ci prétendent servir l'intérêt général, ou tout au moins celui de l'appareil sécuritaire du régime en place, on parlera de diplomatie "parallèle » ${ }^{13}$. L'usage de leurs relations privées par les agents de l'État, à des fins personnelles, est moins directement assimilable à la diplomatie et relève plutôt du clientélisme.

\section{Éléments de définition et esquisse de typologie des modes de privatisation de la politique étrangère}

La notion de "privatisation de la politique étrangère » est polysémique : elle recouvre des phénomènes variés, qui mobilisent des acteurs aux objectifs divergents. Avant de proposer une esquisse de typologie de ces formes d'action internationale nonconventionnelle, il s'agit donc de définir aussi rigoureusement que possible les termes employés, en justifiant le recours au vocable de la diplomatie pour s'en référer à des mobilisations politiques et à des politiques publiques qui, à première vue, ne relèvent pas de son champ d'activité. 


\section{Éléments de définition}

7 De manière extensive, nous définirons ici la politique étrangère comme le projet politique d'une collectivité ou d'une institution, à travers lequel celles-ci cherchent à configurer à leur avantage leur environnement international. La notion de "diplomatie", pour sa part, fait ici référence à la mise en œuvre concrète de la politique étrangère, à travers un système d'actions à voies multiples ${ }^{14}$. Cette définition présente le mérite de sa neutralité: elle rompt avec le cadre d'interprétation " réaliste », qui a longtemps fait de la diplomatie un monopole des États, alors que tout groupe social peut se donner les moyens de conduire une politique étrangère. Le caractère extensif de cette définition pourra heurter les plus "réalistes » des internationalistes et, surtout, les professionnels de la diplomatie, qui pourront souligner le manque de légitimité des diplomates officieux et leurs ressources limitées, obérant leur pouvoir de décision. En dépit des difficultés qu'ils rencontrent pour réunir l'ensemble des attributs de la fonction diplomatique (le pouvoir tribunitien, les capacités de lobbying, les ressources financières et les moyens de pression militaires), ces acteurs peuvent pourtant se doter de projets internationaux et des moyens - même limités - de leur mise en œuvre. La distinction avec les diplomates officiels est ici de degré, plutôt que de nature.

8 Un autre point pourra prêter à confusion : l'articulation entre les pratiques violentes (guerre, terrorisme...) et la diplomatie, dans son volet étatique ou sous ses déclinaisons non-étatiques. Dans la théorie clausewitzienne qui a structuré les représentations de la politique étrangère et de la conflictualité au $\mathrm{xX}^{\mathrm{e}}$ siècle, la guerre constitue la continuation de la politique par d'autres moyens ; elle n'est donc plus de la diplomatie. Mais l'aphorisme clausewitzien ne vaut que pour les conflits interétatiques de grande intensité, mettant en prise des armées régulières. Dans certaines situations conflictuelles contemporaines, comme dans la rivalité indo-pakistanaise, le recours à des formes de conflictualité indirectes et limitées, sous-traitées à des acteurs privés, vient remettre en cause ces conceptions modernes du conflit et relève moins de la guerre que de la diplomatie, même s'il s'agit ici d'une diplomatie " parallèle », c'est-àdire occulte et déléguée à des acteurs irréguliers, non reconnus par le droit international. Comme on le verra plus bas, l'instrumentalisation des groupes insurgés indiens de la part du Pakistan vise ainsi à faire pression sur l'Inde pour l'inciter à la négociation sur le statut du Cachemire et pour l'affaiblir économiquement. Certes, des groupes jihadistes comme le Lashkare Tayyeba n'ont guère d'ambition diplomatique. Leurs mentors étatiques, en revanche, ont un agenda limpide et quasi inamovible, focalisé sur l'obtention de concessions de la part de l'Inde sur le statut du Cachemire. La réticence des autorités pakistanaises, civiles comme militaires, à engager une véritable guerre privatisée au Cachemire se trouve confirmée a contrario par la stratégie pakistanaise en situation de conflit ouvert avec l'Inde. Ainsi, en 1965, 1971 et 1999, c'est l'armée régulière qui s'est trouvée en première ligne (y compris, comme à Kargil en 1999, quand les militaires étaient revêtus des oripeaux des mujahidin ${ }^{15}$ ). La politique afghane du Pakistan offre en revanche une illustration des modes de privatisation de la guerre, dans la mesure où le recours aux jihadistes visait à instaurer un régime allié à Kaboul, plutôt qu'à faire pression sur les dirigeants afghans en vue de parvenir à une résolution des contentieux entre les deux États. 
9 Si la diplomatie est l'instrument des politiques étrangères, publiques ou privées, elle n'est pas neutre dans son déploiement : « l'instrument n'est jamais passif; il a sa propre dynamique, qui vient modifier les perceptions et les choix initiaux ${ }^{16} »$. C'est ce qu'illustre de manière exemplaire la trajectoire diplomatique du Pakistan, aujourd'hui confronté à l'effet de boomerang de sa diplomatie parallèle indienne et de sa privatisation de la guerre en Afghanistan.

10 La notion de «modes populaires d'action diplomatique » appelle elle aussi certaines précisions pour se prêter à un usage rigoureux. Il s'agit des mobilisations politiques ${ }^{17}$ à travers lesquelles les acteurs non-étatiques se mettent en scène dans l'espace public international. L'usage du terme "populaire » pourra ici prêter à confusion, dans la mesure où les diplomates privés sont souvent doublement minoritaires (du fait de leur appartenance à un groupe social numériquement réduit et de leur légitimité contestée au sein de ce groupe). Sous son acception sociologique (en relation au statut social des acteurs et non plus à leur légitimité politique), la dimension populaire des diplomaties privées apparaît tout aussi contestable. Les diplomates officieux sont généralement issus des classes supérieures et bénéficient, de par leur éducation et leurs réseaux sociaux, d'un accès aux médias globaux. Mais chez François Constantin, à qui l'on doit cette notion ${ }^{18}$, le caractère " populaire " des mobilisations en question procède moins $\mathrm{du}$ statut social des acteurs que de leur façon de concevoir et de mener leurs projets politiques "par le bas ", hors de la sphère étatique ou à ses marges. Cette notion fait explicitement référence à celle de "modes populaires d'action politique » développée par Jean-François Bayart pour penser les formes d'action collective et les registres d'énonciation du politique des groupes dominés en Afrique subsaharienne ${ }^{19}$. Pour éviter tout malentendu, rappelons que cette conception du politique "par le bas" n'idéalise aucunement la «société civile " au détriment de l'État (pas plus, d'ailleurs, qu'elle ne postule leur opposition) et ne fait que replacer au cœur de l'analyse du pouvoir les groupes rendus subalternes par leur exclusion des processus décisionnels officiels. Appliquée aux modes d'action diplomatique, l'épithète "populaire » fait donc référence aux registres d'action collective de groupes sociaux écartés de la conduite et du commentaire légitime des affaires internationales par l'aspiration des États au monopole de la représentation des individus.

11 La notion de diplomatie identitaire à laquelle nous aurons également recours ici mérite à son tour d'être explicitée. Il s'agit des dispositifs publicitaires que déploient les associations de défense des victimes de violence politique ${ }^{20}$ et les organisations nationalistes sur la scène internationale, afin d'assurer la protection ou l'indemnisation de ces peuples victimisés et d'obtenir une reconnaissance de la part de la " communauté internationale ». Ces acteurs exercent une fonction diplomatique dans la mesure où ils entretiennent des relations fréquentes et diversifiées avec des acteurs étrangers, étatiques ou intergouvernementaux, qu'ils s'efforcent de rallier à leur projet identitaire (accès à l'autonomie ou à la souveraineté, protection d'une identité culturelle prétendument menacée par un groupe dominant et culturellement différencié).

\section{Esquisse de typologie}

12 Toute tentative de typologie des formes non-conventionnelles d'action diplomatique se heurte au caractère multifonctionnel et mouvant des réseaux qui les -mettent en 
œuvre. Ces réseaux peuvent en effet intervenir simultanément dans plusieurs domaines et ils font souvent preuve d'une remarquable adaptabilité à leur environnement national et international ${ }^{21}$. Malgré les limites inhérentes à l'exercice typologique, dans le cas qui nous occupe ici, il n'est pas inutile de chercher à catégoriser les multiples occurrences du phénomène, ne serait-ce que pour souligner sa diversité, en particulier en ce qui concerne le rapport des diplomates officieux à l'État.

13 La typologie que nous proposons ici (tableau ci-dessous) repose sur une catégorisation des formes non-conventionnelles d'action diplomatique en fonction de leur degré d'autonomie par rapport aux États, qui en dépit de la concurrence de leurs sociétés demeurent la "pièce maîtresse du système diplomatique » contemporain ${ }^{22}$. Du plus privé au plus étatique, on trouve ainsi - en Asie du Sud, tout au moins - deux grandes déclinaisons du phénomène, qui se subdivisent en plusieurs sous-catégories. À la première extrémité de ce continuum, figurent d'abord des « modes populaires d'action diplomatique » qui ont émergé en marge et à l'encontre de l'action diplomatique officielle, à l'initiative de pacifistes œuvrant dans le domaine de la diplomatie informelle (track III diplomacy). Les pratiques de sous-traitance stratégique, qui incluent les diplomaties parallèles et les phénomènes de privatisation de la guerre, se situent à l'autre extrémité de ce continuum. Les diplomaties identitaires des organisations nationalistes en quête de reconnaissance internationale, qui constituent elles aussi un "mode populaire d'action diplomatique», -occupent pour leur part une position médiane sur ce continuum autonomie/ dépendance vis-à-vis des États, dans la mesure où elle sont l'initiative de groupes privés en quête de soutiens étatiques.

Tableau 1

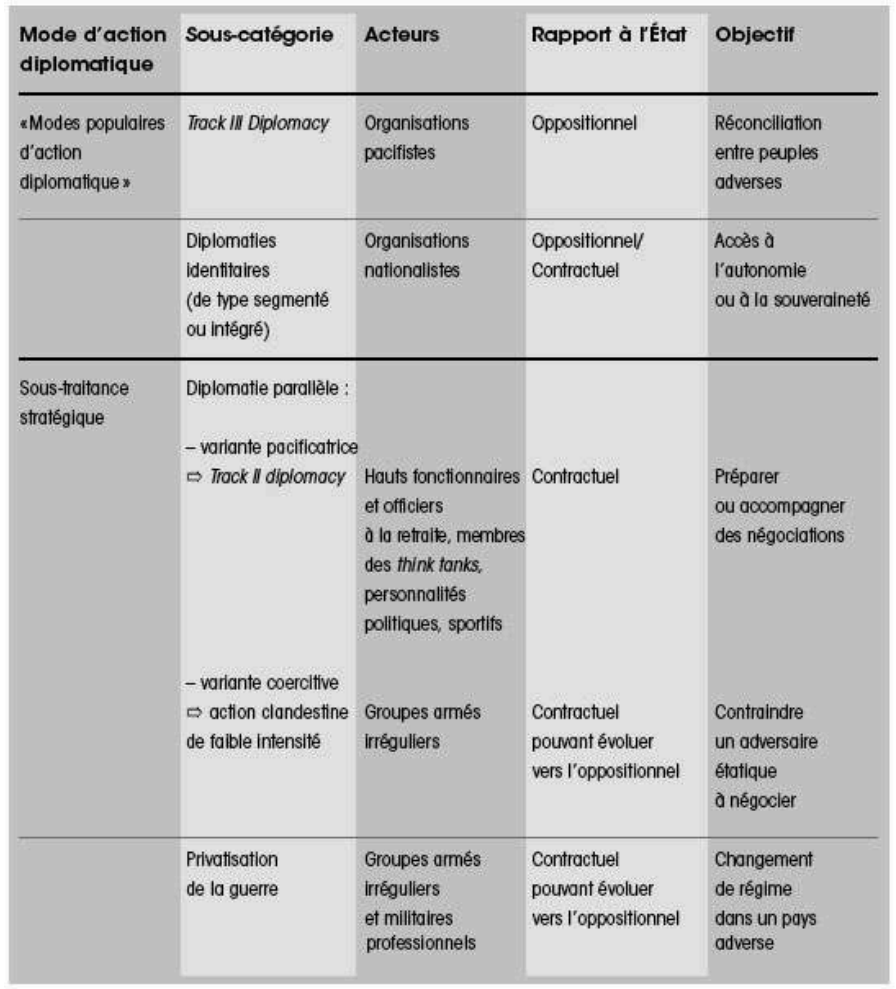

Typologie des formes non-conventionnelles d'action diplomatique en Asie du Sud 
Comme le suggère cet essai de typologie, qui demanderait à être ajusté à la lumière des manifestations du phénomène dans d'autres régions du monde, les partenariats qui s'instaurent dans le champ de la diplomatie ou de la guerre entre les acteurs étatiques et non-étatiques sont souvent instables. La collusion n'aboutit pas nécessairement à la symbiose $^{23}$ et peut, surtout lorsqu'il est question de violence, déboucher sur l'affrontement. Le recours des acteurs privés à la violence peut être toléré, voire encouragé par l'État, ou tout au moins par certains de ses agents, pendant une certaine durée. Mais, sous l'effet de contraintes intérieures ou de pressions extérieures, les patrons étatiques des groupes armés irréguliers sont souvent amenés à prendre leurs distances avec leurs anciens protégés ou au contraire à mieux les encadrer, ce changement de stratégie ne laissant jamais indifférentes les organisations miliciennes, qui peuvent alors se retourner contre leurs anciens mentors étatiques. C'est ce qu'illustre l'exemple pakistanais, mais aussi celui de la politique afghane des États-Unis des années 1980-1990, la sous-traitance de la guerre à l'extérieur ayant dans les deux cas abouti à l'éclosion de conflits inédits sur le territoire national. Si l'État peut temporairement sortir victorieux de ses guerres par procuration, l'histoire récente de l'Asie du Sud indique ainsi qu'aux guerres privatisées succède rarement une paix durable, l'État peinant à retrouver le monopole de la violence légitime après l'avoir partagé avec ses partenaires privés. Les risques de dérive qui nous semblent inhérents à la privatisation de la guerre ne sont pas absents de l'exercice coercitif de la diplomatie parallèle, qui peut rapidement échapper au contrôle de ses acteurs étatiques pour évoluer vers une forme de conflit de plus grande intensité que celle anticipée. À ce titre, la frontière entre les formes coercitives de diplomatie parallèle et les conflits privatisés n'est pas intangible, la première pouvant déboucher sur une escalade militaire alors que la seconde tend à décroître d'intensité lorsque l'État prend la décision et parvient effectivement à retrouver le monopole de la violence légitime.

\section{Les « modes populaires d'action diplomatique » d'Asie du Sud}

Les diplomaties privées d'Asie du Sud présentent des figures contrastées. On trouve en effet, d'un côté, des mouvements nationalistes en quête de rayonnement international et, de l'autre, des mouvements pacifistes œuvrant à la réconciliation des sociétés indienne et pakistanaise par la promotion des contacts entre les deux peuples (people to people contact). En dépit de leurs modes opératoires et de leurs objectifs divergents, les diplomates identitaires et les mouvements pacifistes se rejoignent dans le rapport profondément ambivalent qu'ils entretiennent à l'État. Les pacificateurs privés idéalisent la "société civile» au détriment des normes de souveraineté et de territorialité, mais le développement du « people to people contact» en Asie du Sud reste étroitement tributaire de l'évolution des relations interétatiques régionales. Les diplomates identitaires, pour leur part, se sont livrés à un usage stratégique de l'international dans leur lutte contre les autorités de leur État d'attache, mais ils ont dans le même temps recherché le soutien d'acteurs étatiques étrangers, qui se sont parfois efforcés de les satelliser comme dans le cas du Pakistan à l'égard des groupes insurgés afghans, sikhs ou cachemiris. 


\section{Le mouvement pacifiste indo-pakistanais et la « track III Diplomacy »}

Depuis quelques années, on assiste à l'émergence d'un mouvement pacifiste indopakistanais, porté par les « sociétés politiques ${ }^{24}$ » des deux pays et relayé par un réseau transnational de pacifistes diasporiques ${ }^{25}$. Ces acteurs pacifistes, généralement issus des milieux intellectuels (journalistes, écrivains, universitaires...) ont commencé à s'organiser au cours de la première moitié des années $1990^{26}$. Ils se sont d'abord rassemblés au sein du Forum des peuples indo-pakistanais pour la paix et la démocratie (PIPFPD), fondé en 1994, qui œuvre au renforcement des liens transnationaux entre les sociétés indienne et pakistanaise, à travers le "people to people contact». Mais ce sont surtout les essais nucléaires de l'Inde puis du Pakistan, survenus en mai 1998, qui ont fait de ce mouvement pacifiste transnational une force avec laquelle les gouvernements de la région doivent désormais compter. Ces essais nucléaires ont en effet incité les militant(e)s écologistes et féministes ainsi que les défenseurs des droits de l'homme à s'engager sur la voie du pacifisme, tout en conduisant des citoyens sans expérience militante préalable à rejoindre les organisations anti-nucléaires apparues à cette occasion, telles que le Movement in India for Nuclear Disarmament (MIND), le Parmanu Bomb Virodhi Andolan (Mouvement contre les armes nucléaires, PBVA) ou les South Asians Againt Nukes.

Bien que ce mouvement pacifiste transnational ait un agenda ambitieux (contraindre les États de la région à ouvrir leurs frontières, à réduire leurs dépenses de défense voire à renoncer à l'arme nucléaire) son audience et sa capacité -d'influence demeurent limitées. Ces modes populaires d'action diplomatique ne concernent qu'une minorité d'intellectuels urbains ${ }^{27}$, dont la visibilité médiatique est inversement proportionnelle à leur poids politique. En s'institutionnalisant à travers des actions de protestation routinières, telles que la commémoration des bombardements de Hiroshima et Nagasaki, ce mouvement contestataire court également un risque : celui de perdre sa capacité mobilisatrice et sa force d'innovation. Ce danger est bien identifié par certains pacifistes indiens, qui plaident pour une revivification du mouvement anti-nucléaire régional à travers l'élargissement de ses préoccupations et la formulation de cadres d'interprétation alternatifs, venant remettre en cause la nucléarisation des États de la région mais aussi, plus fondamentalement, la fétichisation des sciences et des technologies de pointe qui a rendu possible l'avènement des nationalismes nucléaires indien et pakistanais ${ }^{28}$.

$\mathrm{Si}$, par ses accents contestataires et ses connexions transnationales, ce mouvement pacifiste paraît remettre en cause la donne politique régionale, il a dû compter avec la résilience des acteurs étatiques dans le système diplomatique sud-asiatique. Le " people to people contact » reste tributaire de la politique de visas des États de la région et ces derniers ont parfois laissé agir les diplomates officieux du Track III pour tester des idées ou explorer des pistes de négociation sans pour autant apparaître soutenir ces initiatives, comme dans le cas du Track II, patronné voire impulsé par les États. 


\section{Les diplomaties identitaires des minorités contestataires d'Inde et du Pakistan}

19 Animée par des acteurs privés aspirant à la reconnaissance internationale, la diplomatie identitaire témoigne de la capacité des entrepreneurs de l'identité à s'échapper - au moins temporairement - du local pour se mobiliser sur la scène internationale en quête de publicité, de soutiens et de financements extérieurs. Comme nous l'évoquions en introduction, les premiers mouvements sociaux transnationaux (abolitionniste, féministe et socialiste) ont vu le jour dès le XIX`siècle. Mais ces mobilisations transnationales se sont structurées autour d'idéologies universalistes qui prétendaient transcender les barrières culturelles. $\mathrm{Au}$ contraire, la diplomatie identitaire repose sur la médiatisation, à des fins défensives, d'une identité culturelle menacée. La première occurrence de ces diplomaties de l'identité fut donc selon nous la mobilisation transnationale de la diaspora juive en faveur des juifs d'Europe, au cours des années 1930 . Cette "diplomatie sans souveraineté ${ }^{29}$ ", motivée par un projet solidariste, s'adressait déjà aux États, aux acteurs non-étatiques (la Croix-Rouge) et aux organisations internationales émergentes. Elle se trouva formalisée et encadrée par le World Jewish Congress, qui dès 1941 animait un réseau transnational couvrant l'Europe, l'Amérique du Nord, la Rhodésie et le Japon.

Au cours de la guerre froide, ces initiatives diplomatiques officieuses mises au service de compatriotes ou de coreligionnaires menacés par leur propre État se sont multipliées. Les minorités contestataires du sous-continent indien (les sikhs, les Cachemiris, les Nagas ou encore les Tamouls sri-lankais) ont tous recouru à cet usage stratégique de l'international dans leurs luttes de libération nationale, mais selon des modalités très différentes. L'étude comparée que nous avons menée sur les dispositifs publicitaires des organisations nationalistes sikhes et mohajires sur la scène internationale ${ }^{30}$ nous a d'ores et déjà permis d'identifier deux formes de diplomatie identitaire: une variante segmentée et une variante intégrée. Les organisations nationalistes sikhes qui se sont mobilisées sur la scène internationale au lendemain des événements de 1984 (attaque de l'armée indienne contre le Temple d'or d'Amritsar, assassinat du Premier ministre Indira Gandhi par ses gardes du corps sikhs, pogroms anti-sikhs, à la suite de cet assassinat, dans tout le Nord de l'Inde) ont été victimes d'un phénomène de scissiparité qui a abouti à la prolifération de groupuscules rivaux. Ces derniers se sont révélés incapables d'acquérir le monopole de la parole légitime (auprès des sikhs eux-mêmes mais aussi de la "communauté internationale ») sur la «nation sikhe » et son destin. À l'opposé de cette tendance au factionnalisme et à la dissonance diplomatique, la dynamique de transnationalisation du Mohajir Qaumi Movement pakistanais (MQM), qui depuis sa fondation en 1984 s'est fait le porte-parole de la communauté imaginée des «Mohajirs " ${ }^{31}$, s'est singularisée par son centralisme autoritaire, à l'opposé de la logique de fonctionnement habituellement prêtée aux réseaux transnationaux. En dépit de son exil à Londres en 1992, le leader du MQM, Altaf Hussain, est parvenu à maintenir un contrôle étroit sur les unités du parti au Pakistan et à l'étranger (Grande-Bretagne, Canada, États-Unis, pays du Golfe, Afrique du Sud...). Le MQM a bien connu quelques vicissitudes au cours des années 1990, à la suite de l'apparition d'une faction concurrente à Karachi (le MQM Haqiqi, ou « véritable »), en raison des velléités réformatrices des militants canadiens ou encore des pressions des cadres et des militants les plus radicaux du parti, répartis entre la Grande-Bretagne et 
le Pakistan, et un moment tentés de déclencher une lutte armée pour l'indépendance de Karachi. Mais contre toute attente, pour un parti fondé par des amateurs en politique ${ }^{32}$, le MQM a surmonté toutes ses crises internes et l'autorité charismatique d'Altaf Hussain demeure incontestée parmi les cadres et les militants du parti, qui se vivent comme les murids de la tradition soufie ${ }^{333}$, en dépit du discours résolument laïc de leur chef.

21 Si la culture politique des diplomates identitaires, ici entendue comme leur " grammaire de l'autorité et du consentement ${ }^{34}$ », fournit l'une des clés d'explication de leur succès ou de leur échec à s'ériger en porte-parole légitime de leur « communauté » sur la scène internationale, le positionnement stratégique de ces acteurs a joué un rôle tout aussi décisif. Par cette formule, nous entendons ici la finalité que ces organisations nationalistes opérant à distance ont cherché à donner à leurs opérations -publicitaires internationales. Dans le cas sikh, les diplomates identitaires expatriés ont initialement concentré leurs revendications sur l'accès du Pendjab à la souveraineté, au nom de leurs compatriotes prétendument bâillonnés par la répression indienne. Mais après avoir consacré l'essentiel de leurs activités à la défense des droits politiques de la minorité sikhe en Inde, ces nationalistes se sont progressivement recentrés sur la défense des droits culturels des minorités sikhes de l'étranger ${ }^{35}$. Le discours nationaliste sikh, y compris sous ses formes les plus radicales, est alors devenu une idéologie de l'exil, c'est-à-dire un mode de gestion des difficultés économiques, politiques et psychologiques inhérentes à l'émigration. Cette appropriation du nationalisme radical sikh par des expatriés en quête de reconnaissance dans leur milieu d'accueil a contribué au discrédit de la cause indépendantiste au Pendjab, où elle est désormais associée aux politiques identitaires de la diaspora, parfois accusée de déstabiliser inconsidérément la province. Au contraire, le MQM n'a jamais dévié dans ses priorités et ses dirigeants ont toujours eu les yeux rivés sur le Pakistan, et plus précisément sur les grands centres urbains du Sind (Karachi, Hyderabad et dans une moindre mesure Sukkur), où se concentre la population ourdouphone imaginée dans les années 1980 comme les «Mohajirs ». De surcroît, Altaf Hussain a mis a profit les technologies de l'information et de la communication (téléphonie mobile et Internet) pour instaurer une relation directe avec ses adeptes dispersés à travers le monde, notamment au moyen de conférences téléphoniques destinées à compenser l'éloignement géographique du leader par un sentiment de plus grande accessibilité.

Les modes d'organisation et l'orientation stratégique des diplomates identitaires permettent d'expliquer leur succès ou leur échec au sein de la communauté qu'ils s'attachent à protéger, mais ne suffisent pas à expliquer les réponses de leur auditoire à l'extérieur de cette communauté. Ici encore, la comparaison entre les diplomaties identitaires des sikhs et des Mohajirs est riche d'enseignements, mais cette fois en raison de leurs analogies plutôt que de leurs contrastes. Dans les deux cas, c'est aux États-Unis que les diplomates identitaires ont remporté leurs principaux succès diplomatiques, qui se sont matérialisés par des soutiens parlementaires mais aussi par une reconnaissance de la part de la Maison Blanche en tant qu'organisation légitime (dans le cas du MQM) ou par un soutien à la cause des droits de l'homme dans le souscontinent indien (dans les deux cas). Les autorités britanniques ont également répondu favorablement à la diplomatie identitaire du MQM, en accordant la citoyenneté britannique à Altaf Hussain et en l'autorisant à poursuivre ses activités politiques en délocalisant le centre de commandement de son parti à Londres. Les militants sécessionnistes sikhs peuvent pour leur part se féliciter d'avoir longtemps retardé la 
signature, entre Londres et New Delhi, d'un accord de coopération dans le domaine de la lutte anti-terroriste, autorisant sous certaines conditions l'extradition des personnes suspectées d'implication dans des actes de terrorisme en Inde. Et les militants du Khalistan Council, proches de la principale force de guérilla sikhe, la Khalistan Commando Force, sont parvenus à obtenir (et à conserver) le soutien actif d'un élu travailliste de la banlieue ouest de Londres, John McDonnell, qui s'est fait le porteparole de ce groupe, converti en lobby culturel (les «Punjabis in Britain») à la fin des années 1990.

Au Canada, en revanche, les sikhs et les Mohajirs ont essuyé une série d'échecs diplomatiques cinglants, qui se sont matérialisés par une étroite coopération policière avec les autorités indiennes et par une relation distante avec le MQM, dont les diplomates professionnels canadiens rencontrés au cours de nos recherches ont toujours refusé d'admettre la présence sur le sol national. Cette réponse singulière du Canada aux diplomaties identitaires de ses minorités, dès lors qu'elles prennent pour cible des partenaires du pays, s'explique en grande partie par un facteur conjoncturel : l'attentat perpétré en 1985 contre un appareil d'Air India par une organisation fondamentaliste sikhe basée à Vancouver, le Babbar Khalsa International (BKI). Les diplomates canadiens restent profondément marqués par cet épisode et par les tensions qui ont émaillé les relations d'Ottawa avec New Delhi sur la question sikhe tout au long des années 1980. Audelà des facteurs structurels profitant aux diplomates identitaires dans leurs États de résidence (c'est-à-dire des opportunités politiques ouvertes par le poids électoral des minorités dans certaines circonscriptions, par le mode de financement des candidats, par la légitimité des groupes de pression "ethniques», par le multiculturalisme...), il s'agit donc de prêter attention aux effets de contingence imposés par la conjoncture politique de chaque État d'accueil des minorités agissantes, qui peuvent s'avérer plus déterminants encore que la variable lourde des opportunités politiques pour la réception des discours nationalistes contestataires ${ }^{36}$.

\section{Les pratiques de sous-traitance stratégique en Asie du Sud}

Si les modes populaires d'action diplomatique battent en brèche le monopole du Prince sur la politique étrangère, ce dernier peut aussi chercher à tirer parti des relations internationales de ses sujets, au nom de la «raison d'État» ou pour des motifs plus personnels. En Inde et au Pakistan, ce volet étatique du phénomène de privatisation de la politique étrangère, que nous qualifierons ici de "sous-traitance stratégique ", se manifeste à travers deux modalités : la diplomatie parallèle (qui comporte un volet pacificateur et un volet coercitif) et la privatisation de la guerre. Ces deux pratiques de la sous-traitance stratégique et leurs déclinaisons peuvent s'alterner ou au contraire se combiner, comme l'illustre l'évolution récente de la politique étrangère pakistanaise.

\section{Sous-traiter la paix : l'exemple de la « diplomatie du cricket »}

Si la Track III Diplomacy s'est développée en marge, voire à l'encontre des États de la région, tel n'est pas le cas de la Track II Diplomacy, dans laquelle l'Inde et le Pakistan ont chacun trouvé un instrument diplomatique parallèle contribuant à réduire les tensions interétatiques en période de crise, et préparant ou accompagnant les discussions 
officielles en période de normalisation. Dans le cadre de cette diplomatie parallèle à vocation pacificatrice, les gouvernements indiens et pakistanais ont principalement eu recours à d'anciens agents de la fonction publique (ex-diplomates et militaires à la retraite) et à des responsables politiques issus de partis minoritaires (tels que le Jamiate Ulema-e Islam de Fazl-ur Rehman, dans le cas pakistanais ${ }^{37}$ ), mais ils se sont aussi tournés vers les milieux sportifs.

La «diplomatie du cricket» qui s'est mise en place dans le sous-continent indien au cours des années 1980 illustre bien la complexité de ces phénomènes de diplomatie parallèle, qui peuvent se déployer dans le sens de la paix ou, comme nous le verrons plus loin, de la guerre. Cette diplomatie sportive ${ }^{38}$, destinée à réduire les tensions indopakistanaises, a d'abord été impulsée par les États. Depuis la seconde moitié des années 1980, les dirigeants indiens et pakistanais ont pris conscience des vertus normalisatrices des rencontres sportives entre leurs deux pays. La tournée de l'équipe pakistanaise en Inde, en 1999, a été décidée par le Premier ministre de l'époque, Nawaz Sharif. De la même manière, l'équipe indienne s'est trouvée mandatée par le Premier ministre Atal Behari Vajpayee pour « conquérir les cœurs et les esprits » au Pakistan en 2004. Dans les deux cas, c'est donc le chef de gouvernement qui a endossé la responsabilité d'envoyer l'équipe nationale dans le pays adverse, avec une mission diplomatique explicite, résumée par le capitaine de l'équipe pakistanaise, Wasim Akram, peu avant sa tournée en Inde en 1999: « Nous allons là-bas pour contribuer à une amélioration des relations entre nos deux pays ${ }^{39} "$. On peut donc bien parler ici de sous-traitance diplomatique, les joueurs de cricket se voyant confier une mission d'intérêt général par leur État d'attache. Cette mission consiste moins à pacifier les relations indo-pakistanaises qu'à accompagner les négociations entre les deux États. En ce sens, la diplomatie du cricket n'est pas un instrument de peace-building mais une mesure de confiance sociétale, destinée à préparer les esprits à la tenue d'un nouveau round de négociation. Cette diplomatie, déléguée à des acteurs privés mais étroitement surveillée par des acteurs étatiques, constitue donc un préalable et non un substitut au dialogue entre les deux États rivaux.

Si la diplomatie du cricket sert des stratégies étatiques, elle n'y est pas entièrement réductible. Par bien des côtés, cette diplomatie sportive relève aussi de logiques strictement privées. Celles-ci sont de deux natures: identitaire et mercantile. Les matchs de cricket entre l'Inde et le Pakistan donnent aujourd'hui l'occasion aux sociétés indienne et pakistanaise de renouer des liens distendus par la Partition puis par les guerres à répétition qui ont marqué l'histoire des relations indo--pakistanaises. À leur échelle individuelle, les « touristes du cricket » et leurs hôtes (parfois bénévoles ${ }^{40}$ ) s'efforcent de surmonter l'animosité qui prévaut entre leurs États pour célébrer un univers de sens partagé. Ceci est particulièrement vrai pour les populations les plus affectées par la Partition, en particulier les sikhs (en Inde) et les Mohajirs (au Pakistan). Ce sont ces populations, gardant des attaches familiales (dans le cas des Mohajirs) ou religieuses et affectives (dans le cas des sikhs) chez « l'ennemi », qui ont soutenu avec le plus de ferveur la diplomatie du cricket, en réservant un accueil chaleureux aux joueurs et aux visiteurs du pays voisin. Or, pour ces populations victimes de la Partition, la diplomatie du cricket est moins synonyme de rapprochement entre l'Inde et le Pakistan que de retrouvailles avec les êtres et les lieux chers restés de l'autre côté de la frontière. Ce rapprochement infra-étatique est aussi sub-national puisqu'il concerne des populations qui, pour une partie d'entre elles tout au moins, se 
reconnaissent dans une identité ethnolinguistique minoritaire (celle des locuteurs de l'ourdou dans le cas des Mohajirs et de leurs relations familiales en Inde, et celle des pendjabiphones dans le cas des sikhs et des Pendjabis pakistanais).

Les élites politiques ne sont pas épargnées par ces émotions transnationales, teintées de nostalgie pour ceux qui ont grandi dans le pays voisin. Ainsi l'un des plus virulents détracteurs des musulmans indiens, Lal Krishna Advani, laissa-t-il échapper son émotion au cours de sa rencontre avec l'équipe de cricket pakistanaise lors de sa tournée en Inde en 1999. Alors ministre de l'Intérieur dans le gouvernement d'Atal Behari Vajpayee, Advani s'était rendu tristement célèbre au début de la décennie en orchestrant la campagne d'agitation qui s'était soldée par la destruction de la mosquée de Babour, à Ayodhya, en 1992. Mais Advani est aussi un Sindi, natif de Karachi, et il conserve de solides liens affectifs avec cette province, aujourd'hui pakistanaise. Au cours de cette rencontre, Advani a donc été conduit à évoquer avec les joueurs pakistanais son enfance passée à Karachi, dans un accès de nostalgie qui a paru d'autant plus sincère aux yeux de ses interlocuteurs qu'il leur semblait relever d'affects privés plutôt que d'une posture publique. Le vieillard qui se remémorait devant eux ses années de jeunesse à la St. Patrick's School était une personne privée, bien plus qu'un représentant de l'État ${ }^{41}$.

29 Parallèlement à ces logiques identitaires infra-étatiques, la diplomatie du cricket s'articule à des logiques mercantiles. Cela est particulièrement vrai au Pakistan. Le Pakistan Cricket Board (PCB), côté comme une entreprise privée à la Bourse de Karachi, fait face à un déficit chronique qui s'est aggravé depuis 2001, à la suite du boycottage du pays - jugé peu sûr ${ }^{42}$ par de nombreuses équipes étrangères. Entre 2001 et 2004, la seule équipe étrangère à avoir accepté de disputer un match au Pakistan fut celle du Bangladesh. Or, les rencontres avec l'équipe bangladaise sont les moins lucratifs des matchs internationaux disputés à domicile par l'équipe pakistanaise, dans la mesure où elles font l'objet de faibles droits de retransmission télévisuelle. Dans ce contexte financier précaire, les tournées de l'équipe indienne constituent une véritable aubaine pour le PCB et ses actionnaires. Peu avant la tournée de l'équipe indienne au Pakistan, en 2004, le porte-parole du PCB a ainsi confié à la presse que cette visite devait rapporter plus de 20 millions de dollars à la fédération (21 millions de dollars de droits de retransmission et 1,25 million de dollars de la vente des tickets ${ }^{43}$ ).

Ces logiques mercantiles sous-tendant la diplomatie du cricket viennent aussi la perturber. Si les entrepreneurs pakistanais du cricket soutiennent aujourd'hui la reprise des échanges sportifs avec l'Inde pour maximiser leurs profits, ils se trouvent confrontés aux résistances de leurs concurrents indiens, relayées dans la presse nationale par les commentateurs sportifs ou les simples aficionados du sport. En 2003, une lectrice du site nationaliste hindou Vigilonline s'indignait ainsi de l'entrisme des entreprises pakistanaises sur le marché indien de la rediffusion sportive: «Il est regrettable que nous ayons engagé un processus par lequel les entreprises pakistanaises se verront autorisées à participer et à avoir accès à nos marchés, à travers des matchs largement rediffusés. La bonne volonté est une chose, l'accès aux marchés et aux consommateurs indiens en est une autre - l'Inde s'apprête ici à renoncer à un avantage stratégique ». On notera que cette lectrice énonce son propos mercantile dans l'idiome de la sécurité nationale, les arguments qu'elle apporte dans la suite de son texte pour justifier une suspension des échanges sportifs entre les deux pays étant tous de nature stratégique (persistance du soutien pakistanais aux troubles du Cachemire et 
$\mathrm{du}$ Nord-Est, notamment) ${ }^{44}$. Cet argumentaire suggère que, si les États indien et pakistanais sont aujourd'hui déterminés à faire un usage diplomatique des rencontres sportives entre leurs sociétés, certains acteurs sociaux peuvent pour leur part être tentés de s'opposer à ces rencontres, en s'appropriant alors le discours stratégique de l'État pour défendre leurs intérêts économiques.

31 L'irréductibilité du cricket indo-pakistanais à ses usages politiques et diplomatiques se trouve en dernier lieu attestée par les vitupérations des plus ardents défenseurs de ce sport, qui s'indignent de sa « dérive politicienne ». L'un de ces commentateurs sportifs, écrivant au lendemain de la tournée de l'équipe indienne au Pakistan, début 2006, estimait ainsi que "croire que les jeux sont plus que des jeux et qu'ils symbolisent quelque chose de beaucoup plus profond et radical est une absurdité ». Pour cet auteur, la « diplomatie du cricket » pêche par son romantisme et repose sur « une idée jaillie du cerveau de hippies paresseux » ${ }^{45}$. Pour ces aficionados, la dépolitisation du cricket indo-pakistanais qu'a semblé attester cette récente tournée ${ }^{46}$ est donc une heureuse nouvelle, signant le triomphe du ludique sur le politique.

\section{Sous-traiter la guerre : la politique afghane du Pakistan}

32 La privatisation de la guerre est consubstantielle à la formation de l'État pakistanais ${ }^{47}$, dont les élites politiques ont cherché à sous-traiter leurs conflits extérieurs dès les premiers mois de l'indépendance. Au cours de l'automne 1947, des milliers de miliciens pachtounes déferlèrent de la Province de la frontière du Nord-Ouest (pakistanaise) sur le Cachemire (alors intégralement indien). Islamabad dénia toute implication dans cette invasion, présentée comme spontanée. Les témoignages des officiers indiens ${ }^{48}$ et surtout pakistanais impliqués dans cette première guerre indo-pakistanaise viennent cependant démentir cette thèse officielle. Le principal responsable pakistanais de l'opération "Gulmarg", le général Akbar Khan, alors directeur du programme d'armement et d'équipement à l'état-major de l'armée de terre, confirme ainsi que des officiers des trois armes et des services de renseignement furent impliqués dans la planification et dans l'exécution de cette campagne, qui sur le terrain mobilisa les lashkars pachtounes (épaulés et commandés par des militaires professionnels) mais aussi des vétérans de l'Indian National Army (INA) et des membres de la Muslim Guard, la milice de la Ligue musulmane. Le témoignage de Khan confirme également l'implication du Premier ministre de l'époque, Liaqat Ali Khan, même s'il reste évasif sur le rôle de Jinnah dans l'affaire ${ }^{49}$, aujourd'hui établi ${ }^{50}$.

En recourant aux milices pachtounes pour envahir le territoire contesté du Cachemire, les élites civiles et militaires de l'État pakistanais naissant se sont inspirées de la politique sécuritaire du British $R a j$ dans ses marches tribales frontalières de l'Afghanistan. Soucieux de contenir le projet d'accès aux mers chaudes de l'Empire russe, les Britanniques s'engagèrent au XIX* siècle dans un «Grand Jeu » qui les incita à se rallier les tribus pachtounes du côté oriental de la ligne Durand ${ }^{51}$ pour défendre les frontières de leur empire autant que pour influencer le jeu politique afghan. À partir des années 1920, l'État colonial ne se contenta plus de se ménager le soutien des tribus par une politique de récompenses individuelles (le lungi) ou collectives (le muajib) et il commença à utiliser les lashkars pachtounes pour influencer le jeu politique afghan, tout en se réfugiant derrière une dénégation plausible face aux accusations de nuisance des Russes ou des Afghans ${ }^{52}$. En 1924, les Britanniques firent appel aux tribus 
pachtounes du Waziristan pour renverser le roi Amanullah, dont les réformes sociales et les orientations diplomatiques inquiétaient Londres. En 1929, les lashkars des tribus wazir, mohmand, mangal et jadran furent cette fois utilisés par l'État colonial pour renverser le bandit tadjik Bacha-i Saqao, qui avait usurpé le trône du roi Amanullah et s'était montré incapable de rétablir l'ordre dans le pays ${ }^{53}$. C'est de cette expérience de la guerre privatisée qu'a hérité l'État pakistanais, dans lequel l'historien britannique Ian Talbot a à juste titre reconnu un légataire de "l'État sécuritaire britannique ", reposant sur l'exercice indirect du gouvernement à travers des intermédiaires (propriétaires terriens, chefs tribaux et princes) ${ }^{54}$.

Après leur première expérience (restée sans suite, par ses ambitions) de la guerre privatisée au Cachemire, en 1947-1948, les militaires pakistanais ont affiné leurs pratiques de sous-traitance conflictuelle en Afghanistan. Le soutien apporté par le Pakistan aux premiers rebelles islamistes afghans, de 1973 à 1978, relevait de la diplomatie parallèle, dans la mesure où il visait à faire pression sur le régime du Premier ministre Daoud pour l'inciter à retirer son soutien aux irrédentistes pachtounes du Pakistan et à reconnaître la ligne Durand comme frontière officielle entre les deux États. Ce n'est qu'avec l'invasion soviétique de l'Afghanistan, en 1979, que le Pakistan a renoué avec la guerre privatisée, encouragé en cela par les États-Unis, l'Arabie saoudite ou encore la Chine. Dans les années qui suivirent l'invasion, le Pakistan devint le principal point d'entrée des armes et des fonds à destination des mujahidin, qui installèrent leur quartier général à Peshawar. Le soutien de l'armée pakistanaise aux jihadistes afghans et étrangers fut supervisé sur le terrain par l'un des proches du général Zia, le général Fazl-e Haq, alors gouverneur de la Province de la frontière du Nord-Ouest (NWFP). Le chef de l'État pakistanais s'appuya aussi sur les partis islamistes pakistanais, et en particulier sur la Jama'at-e Islami, pour mettre au point sa stratégie de déstabilisation et prêter main-forte aux jihadistes en Afghanistan. Cette implication de la Jama'at dans l'élaboration de la politique étrangère pakistanaise, à partir de 1978, contribua à son internationalisation, en contraignant ses leaders à développer une perspective régionale et globale qui leur faisait jusqu'alors défaut ${ }^{55}$.

La stratégie américano-pakistanaise fut dans un premier temps couronnée de succès : les Soviétiques, vaincus, virent leur prestige international émoussé, et l'Afghanistan semblait alors voué à retourner dans la sphère d'influence occidentale. Mais l'évolution de la scène politico-militaire afghane, dont les jeux ont toujours partiellement échappé au contrôle d'Islamabad, est rapidement venue contredire ses anticipations. Les factions pachtounes soutenues par Islamabad, en particulier le Hezb-i Islami de Gulbuddin Hekmatyar, ne sont pas parvenues à s'imposer et l'effondrement de l'État afghan consécutif à la prise du pouvoir par les mujahidin, en 1992, a incité les autorités pakistanaises à se trouver de nouveaux alliés dans le pays. C'est avec cet objectif, et celui de rétablir les relations commerciales entre le Pakistan, l'Iran et l'Asie -centrale, que le ministre de l'Intérieur de Benazir Bhutto, le général Naseerullah Khan Babar, un vétéran de la guerre privatisée ${ }^{56}$, a soutenu le mouvement des talibans, avec cette fois l'appui des religieux et du réseau de madrassas du Jamiat-e Ulema-e Islam (JUI). De nouveau, la stratégie sembla s'avérer payante: les talibans parvinrent à conquérir la majeure partie du territoire en l'espace de deux ans (1995-1996) et Islamabad se félicita de l'instauration d'un régime pro-pakistanais à Kaboul. Devant le comportement des talibans, tant dans leur embryon d'État qu'à leur égard, les Pakistanais durent cependant se rendre rapidement à l'évidence : ce mouvement composé en majorité 
d'Afghans nés et éduqués au Pakistan a progressivement acquis une identité et une dynamique propres ${ }^{57}$, à la fois nationaliste (car centrée sur la défense de l'Afghanistan, ici entendu comme la terre des Pachtounes) et internationaliste (à la suite du rapprochement des dirigeants talibans avec Oussama Ben Laden).

Les liens que le général Musharraf fut contraint de rompre avec le régime des talibans, au lendemain des attentats du 11 septembre, s'étaient donc distendus au cours des années précédentes, qui avaient vu les talibans repousser les offres de négociation du Pakistan sur le statut de la ligne Durand puis dynamiter les Bouddhas de Bamyan contre l'avis des services pakistanais. L'ascension politique des Panshiris de l'exAlliance du Nord, connus pour leur proximité avec New Delhi, et le regain d'influence de l'Inde dans le pays et sa région ont cependant incité le Pakistan à renouer des liens avec les talibans, option à laquelle Islamabad n'a pas encore su trouver d'alternative pour s'imposer dans le jeu politique afghan. À court terme, la stratégie pakistanaise semble de nouveau vouée au succès: les talibans gagnent à présent du terrain, le régime du président Karzaï est vacillant et l'accroissement brutal du nombre de victimes auquel on assiste actuellement dans les rangs des troupes de l'OTAN pourrait rapidement inciter les gouvernements occidentaux à retirer leurs troupes, laissant ainsi le champ libre au Pakistan et à ses affidés. Mais tout porte à croire que ce nouvel épisode, dans la saga des guerres privatisées du Pakistan, aboutira in fine à un échec stratégique, dans la mesure où le projet pakistanais de satellisation de Kaboul fait fi de l'autonomie irréductible des acteurs politico-militaires afghans, qui au final retirent toujours les plus gros bénéfices de leur collaboration avec les agents de l'État pakistanais.

Au-delà de leurs échecs répétés en Afghanistan, les dirigeants pakistanais sont aujourd'hui confrontés aux retombées intérieures de leur politique afghane. Le phénomène n'est pas nouveau: dans les années 1980, le pays se trouva inondé d'armes et de drogue, ces flux illicites ayant donné naissance à de nouvelles entreprises criminelles et à des tensions sociales dans les grands centres urbains du pays, en particulier à Karachi ${ }^{58}$. Par comparaison, les troubles du Waziristan, où l'armée pakistanaise se trouve confrontée à la résistance de quelques milliers de combattants jihadistes, pourraient presque sembler insignifiants. Ce mouvement, que les autorités pakistanaises ont finalement décidé de tolérer en passant un accord de -cessez-le-feu avec les insurgés en septembre 2006, porte pourtant en germes la «talibanisation » du Pakistan, ou tout au moins de ses régions pachtounes, qui à terme pourrait bouleverser les équilibres politiques et les relations diplomatiques du pays.

\section{Entre guerre et diplomatie : l'instrumentalisation par le Pakistan des conflits intérieurs de l'Inde}

$\mathrm{Si}$, en Afghanistan, l'armée pakistanaise a mené une véritable guerre privatisée, ses opérations clandestines en Inde relèvent plutôt de la diplomatie parallèle, dans sa variante coercitive. Les accusations de « guerre par procuration » formulées par l'Inde à l'encontre de son rival sont trompeuses: en dépit du soutien moral et militaire qu'elles ont apporté aux groupes insurgés indiens à partir de la seconde moitié des années 1980, les autorités pakistanaises n'ont pas cherché à les utiliser dans un but guerrier, comme elles l'ont fait avec les mujahidin afghans à partir de 1980. L'objectif, ici, n'était pas d'installer un régime allié à Delhi mais plutôt de «saigner » l'Inde pour 
ralentir son décollage économique, de fragiliser son contrat social en entretenant des tensions intercommunautaires nuisant à la cohésion interne du pays mais aussi à son image internationale, et enfin de l'inciter à négocier sur le statut du Cachemire. La pression militaire exercée sur le terrain par les jihadistes devait ainsi se combiner au travail diplomatique mené auprès de la "communauté internationale " pour s'assurer de son soutien, le jour tant attendu où l'Inde finirait par se résigner à engager des négociations sur le territoire contesté.

Si l'on en croit l'un des anciens conseillers de l'ancien Premier ministre Nawaz Sharif, Husain Haqqani, cette politique a été inspirée au général Zia par son maître-espion, le général Akhtar Abdul Rahman, au début des années 1980. Le président pakistanais, déjà engagé dans le conflit afghan, se serait d'abord montré hésitant devant le plan de déstabilisation mis au point par le général Rahman, qui commandait alors le plus important service de renseignement pakistanais, l'Inter Services Intelligence (ISI), fondé au lendemain du premier conflit indo--pakistanais. "Il faut y réfléchir à deux fois ", aurait ainsi répondu le général Zia après un temps de réflexion. La détermination du général Rahman aurait cependant fini par venir à bout des hésitations du chef de l'État pakistanais ${ }^{59}$. En 1984, l'ISI fut chargée de mettre au point une nouvelle stratégie au Cachemire. Contrairement au modèle suivi en Afghanistan, où le service avait été chargé de former directement les mujahidin ( 80000 d'entre eux auraient reçu une formation des officiers du bureau afghan de l'ISI au cours des années $1980{ }^{60}$ ), il fut ici décidé de former des formateurs, qui se chargeraient ensuite de l'encadrement des recrues ${ }^{61}$. L'évolution de la situation sur le terrain, dans la seconde moitié des années 1980, dépassa cependant les attentes des militaires pakistanais, dont la réponse aux insurrections du Pendjab et du Cachemire indiens fut finalement plus réactive que proactive.

Comme le confirment les témoignages que nous avons pu recueillir auprès de militants sécessionnistes sikhs présents au Pakistan dans les années 1980, la coopération entre les services de renseignement pakistanais et les opposants armés au gouvernement indien s'est instaurée de manière progressive et pragmatique et elle n'a jamais été fusionnelle, les agences de renseignement pakistanaises s'étant toujours inquiétées de l'autonomie de ces acteurs non-étatiques alors que ces derniers se méfiaient des velléités de contrôle de leur patron étranger. Il s'agit également de souligner que cette diplomatie parallèle est venue répondre à une demande de soutien diplomatique et logistique de la part d'organisations ou de mouvements sociaux qui lui préexistaient ${ }^{62}$. En ce sens, la diplomatie parallèle pakistanaise - sous son volet coercitif tout au moins - est fondamentalement réactive: elle a en effet répondu aux mouvements transfrontaliers de militants sikhs puis cachemiris préalablement mobilisés, qui ont rejoint le Pakistan de leur propre initiative. Ce n'est que progressivement que l'État pakistanais a choisi de faire de cet état de fait - la présence d'opposants indiens sur son sol - un atout diplomatique. Ainsi, lorsque des centaines de sikhs rejoignirent le Pakistan, dans les semaines qui suivirent l'opération Blue Star ${ }^{63}$, l'ISI s'efforça d'abord de contrôler leurs mouvements sur le territoire pakistanais et de les regrouper géographiquement. La prison de Faislabad fut transformée, dès l'été 1984, en centre de regroupement des militants sikhs, qui ne reçurent aucun entraînement ou appui militaire significatif avant la fin des années 1980 , comme le relate cet ancien militant du Dal Khalsa :

« À cette époque-là [1984], j'étais policier dans le district de Sangrur. J'occupais le rang de head-constable. Après l'opération Blue Star, j'ai compris qu'il ne me serait 
plus possible de vivre en Inde. [...] J'étais déjà en contact avec les militants et j'ai décidé de les rejoindre. Il n'y avait pas de guérilla à ce moment-là, seulement le Dal Khalsa et la [All India Sikh Youth] Federation. En décembre 1984, je décidai finalement de me rendre au Pakistan. Au cours de mes années dans la police, je m'étais trouvé posté dans le district d'Amristar, et je connaissais donc très bien la zone frontalière, si bien que j'ai pu traverser facilement. Si je m'étais fait repérer par la police, ils auraient tiré à vue. Une fois de l'autre côté, les Rangers pakistanais m'ont accueilli et je suis resté quelques jours à Lahore avant d'être conduit à Faislabad. Lorsque je suis arrivé là-bas, il y avait déjà 400 sikhs. Ils ne semblaient pas organisés politiquement et leurs liens avec le gouvernement pakistanais étaient assez limités. La prison avait été transformée en un camp avec toutes les infrastructures requises. L'armée gardait l'endroit mais nous étions traités en invités et en réfugiés. Certains d'entre nous espéraient rentrer au bout de quelques semaines, après que la situation se fut tassée. Mais d'autres voulaient rentrer immédiatement, ce qui nourrissait leur hostilité à l'égard des Pakistanais, qui tardaient à proposer un plan d'action. À ce moment-là, tout le monde parlait de vengeance et ce n'est que plus tard que l'on se mit à parler du Khalistan [l'État souverain revendiqué par les sécessionnistes sikhs depuis les années 1940] ${ }^{64}$ ».

41 Jusqu'à la fin des années 1980, il semble donc que les « services " pakistanais se soient méfiés de ces acteurs non-étatiques aux structures politiques lâches et aux objectifs politiques flous. D'armée de réserve, les militants khalistanis, qui se sont dotés de structures politico-militaires à partir de 1986, sont progressivement devenus des partenaires des services secrets pakistanais, mais ils ont longtemps veillé à ne pas devenir de simples sous-traitants, afin de conserver une certaine autonomie dans leurs actions mais également pour conserver une crédibilité auprès de leur base sociale en Inde. Ainsi, pour un ancien militant de la Khalistan Liberation Force (KLF), qui se rendit également au Pakistan au cours de l'été 1984 :

«Ils avaient leurs propres intérêts. En plus, ils voulaient regrouper les militants afin de mieux les contrôler. Ils ne voulaient pas que nous nous promenions en toute liberté, comme je l'ai moi-même fait. J'étais très atypique pour eux et ils ne savaient pas vraiment comment se comporter à mon égard ${ }^{65}$ " .

42 Les militants khalistanis reprochent également à leurs patrons pakistanais d'avoir empêché l'émergence de structures décisionnelles autonomes au sein de leur mouvement. L'ISI a en effet encouragé les scissions dans leurs rangs et contribué au cloisonnement des multiples groupes armés sikhs apparus à partir de 1986. Ainsi qu'en témoigne un ancien militant de la Khalistan Commando Force (KCF) présent au Pakistan aux côtés de Wasson Singh Zaffarwal ${ }^{66}$ à partir de 1987,

«les Pakistanais nous ont un peu entraînés, mais ils ont refusé que nous nous entraînions par nous-mêmes. Ils ne nous laissaient même pas communiquer entre nous. Ils interdisaient toutes les réunions et empêchaient toute discussion ${ }^{67}$ ".

Des tensions analogues ont émaillé la relation des autorités pakistanaises avec les groupes insurgés du Cachemire. Les services de renseignement pakistanais ont ici cherché à empêcher l'émergence d'un groupe armé trop puissant, qui aurait retiré tout le crédit de la lutte au Cachemire et aurait pu s'en servir pour se faire valoir sur la scène politique pakistanaise. L'ISI a d'abord cherché à affaiblir les indépendantistes laïcs du Jammu Kashmir Liberation Front (JKLF), à l'origine de l'insurrection de 198968 . Après avoir recentré son soutien sur les islamistes du Hizbul Mujahidin, proches de la Jama'at-e Islami pakistanaise, l'ISI a dans un troisième temps patronné les organisations salafistes-jihadistes proches d'al-Qaida (le Lashkar-e Tayyeba et le Jaish-e Mohammad). Disposant d'une assise sociale plus limitée au Cachemire indien que le JKLF et le Hizb, ces groupes étaient plus -facilement -contrôlables et ils avaient le 
mérite de n'entretenir aucun projet politique au Pakistan, alors que les succès militaires du Hizb risquaient de profiter à la Jama'at sur la scène politique pakistanaise. À partir de 1991, l'ISI a également encouragé et aidé les vétérans du jihad afghan à participer au jihad-e kashmir. Pour l'ISI, les jihadistes étrangers, privés de la possibilité de rentrer dans leur pays d'origine, présentaient l'avantage de ne pas hésiter à sacrifier leur vie sur le champ de bataille ${ }^{69}$.

Depuis les attentats du 11 septembre 2001, le Pakistan a été contraint de réduire, ou tout au moins de mieux dissimuler son soutien aux organisations jihadistes opérant en Inde. La répression en demi-teinte qui s'est abattue sur les jihadistes et - surtout - sur les organisations sectaires sunnites ${ }^{70}$, a suffi à provoquer l'ire des milieux religieux radicaux et d'une fraction de l'armée. Ce ressentiment est à l'origine des multiples tentatives d'assassinat dont a été victime le général Musharraf au cours des dernières années, l'une d'entre elles ayant manqué d'aboutir en décembre 2003. Devant ces représailles et les risques de dissension au sein de l'armée, mais aussi convaincu que les mujahidin demeurent un précieux levier de pression sur l'Inde, le général Musharraf s'est jusqu'à présent gardé de mettre un terme au jihad-e kashmir, en se contentant de réduire son intensité.

L'implication très active de l'organisation mère du Lashkar-e Tayyeba (LeT), la Jama'at-ud Da'wa, dans le secours aux victimes du tremblement de terre de décembre 2005, a pour sa part rehaussé l'image des jihadistes auprès de la population pakistanaise, obérant d'autant la capacité de répression des autorités. Cette mise en dépendance de l'État par les groupes jihadistes s'est trouvée confirmée par le Premier ministre Shaukat Aziz au lendemain des attentats de Bombay de juillet 2006, lorsque celui-ci a pris la défense du LeT en affirmant que "nous n'avons aucune preuve de l'implication [du LeT] dans des activités répréhensibles. [...] Et la Jama'at-ud-Da'wa a accompli un travail remarquable au lendemain du tremblement de terre ${ }^{71} »$.

\section{Conclusion}

Les phénomènes de privatisation de la politique étrangère, qui demeurent peu étudiés en relations internationales, invitent à questionner les catégories d'analyse traditionnelles de la discipline. Mais l'on aurait tort de surévaluer la portée corrosive de ces mobilisations politiques transnationales, du point de vue de l'ordre international. Celui-ci demeure dominé par les États, dont les pratiques de soustraitance stratégique ou les ripostes souverainistes aux «modes populaires d'action diplomatique " démontrent les capacités d'adaptation à une donne internationale -mouvante. Les transactions collusives de certains États avec les pacificateurs privés ou avec les nouveaux acteurs du désordre international peuvent aboutir à de véritables partenariats stratégiques (on parlera alors de diplomatie "catalytique $\left.{ }^{72} »\right)$. Ces configurations hybrides du pouvoir sont pourtant rarement stabilisées et se caractérisent plutôt par leur fluidité, traduisant les aspirations divergentes, sinon contradictoires, des parties en présence. Cette instabilité tend à s'accroître lorsque les partenaires sociaux des États délégateurs professent une idéologie dissonant avec celle de leurs mentors étatiques. Le pacifisme affiché par les sportifs professionnels facilite leur recrutement dans le cadre de diplomaties parallèles à vocation pacificatrice et fait de ces acteurs privés des "soldats de la paix " relativement fiables ${ }^{73}$. L'exercice coercitif de la diplomatie parallèle et la privatisation 
de la guerre sont plus délicats, dans la mesure où les partenaires sociaux de l'État sont ici guidés par des idéologies infranationales (ethnonationalisme, sectarisme religieux) ou supranationales (panislamisme) qui diffèrent des nationalismes officiels et qui vont à l'encontre des normes étatiques contemporaines. Les registres de mobilisation de ces acteurs contestataires rendent donc leur contrôle plus difficile et, à terme, exposent les États délégateurs à l'effet de boomerang de leurs diplomaties parallèles et de leurs guerres privatisées.

\section{BIBLIOGRAPHIE}

- Ahmed A. S., « Pukhtun Tribes in the Great Game », in Pakistan Society. Islam, Ethnicity and Leadership in South Asia, Delhi, Oxford University Press, 1988, p. 133-157.

- BECKER A., « Diplomacy without Sover-eignty : the World Jewish Congress Rescue Activities », in S. I. TROEN et B.PINKUS (dir.), National Jewish Solidarity in the Modern Period, Londres, Frank Cass, 1992, p. 343-360.

- Behera N.-C., V. Gunawardena, S. Karadar et R.-A. Mahmood (dir.), People to People Contact in South Asia, Colombo et Delhi, Regional Centre for Strategic Studies et Manohar, 2000.

- BERMAn M. et J. JOHNSON (dir.), Unofficial Diplomats, New York, Columbia University Press, 1977.

- BozARSlan H., La question kurde. États et minorités au Moyen-Orient, Paris, Presses de Sciences Po, 1997.

- BRYSK A., From Tribal Village to Global Village. Indian Rights and International Relations in Latin America, Stanford, Stanford University Press, 2000.

- ConstAntin F., « Affaires de familles et affaires d'État : à propos des modes populaires d'action diplomatique en Afrique orientale », Revue française de science politique, vol. 36, $n^{\circ}$ 4, 1986, p. 672-694.

- CONSTANTIN F., « La privatisation de la politique étrangère : à partir de la scène africaine ", Pouvoirs, n 88, 1999, p. 43-64.

- DORRONSORO G., « La grande illusion : bilan de la politique afghane du Pakistan », Les Études du CERI, $\mathrm{n}^{\circ} 84$, mars 2002.

- foucault M., « Sécurité, territoire et population », Annuaire du Collège de France, 78. année, Histoire des systèmes de pensée, année 1977-1978, 1978, p. 445-449.

- HAQQANI H., Pakistan. Between Mosque and Military, Washington, Carnegie Endowment for International Peace, 2005.

- HівоU B., « Retrait ou redéploiement de -l'État ? ", Critique internationale, n 1, automne 1998, p. $151-168$.

• Hocking B., « Catalytic Diplomacy : Beyond "Newness" and "Decline” ", in J. MELISSEN (dir.), Innovation in Diplomatic Practice, Houndmills, Palgrave, 1999, p. 21-42.

- HUSSAIN R., Pakistan and the Emergence of Islamic Militancy in Afghanistan, Aldershot, Ashgate, 2005. 
- KECK M. et K. SIKKINK, « Historical Precur-sors to Modern Transnational Social Move-ments and Networks », in J. GUIDRY, M. KENNEDY et M. ZALD (dir.), Globalization and Social Movements. Culture, Power, and the Transnational Public Sphere, Ann Arbor, University of Michigan Press, 2000, p. 35-53.

- KHAN S.-M., Cricket, a Bridge of Peace, Karachi, Oxford University Press, 2005.

- KOTHARI S. et Z. MiAn (dir.), Out of the Nuclear Shadow, Delhi et Londres, Lokayan, Rainbow Publishers et Zed Books, 2001.

- NEWsom D. (dir.), Private Diplomacy with the Soviet Union, Lanham, University of America Press, 1987.

- RAMANa M. V et C. RAMmAnohar REDdy (dir.), Prisoners of the Nuclear Dream, Delhi, Orient Longman, 2003.

- тномson J., Mercenaries, Pirates and Sovereigns. State-Building and Extraterritorial Violence in Early Modern Europe, Princeton, Princeton University Press, 1994.

\section{NOTES}

1. M. Foucault, «Sécurité, territoire et population ", Annuaire du Collège de France, $78^{\mathrm{e}}$ année, Histoire des systèmes de pensée, année 1977-1978, 1978, p. 445-449.

2. J. Thomson, Mercenaries, Pirates and Sovereigns. State-Building and Extraterritorial Violence in Early Modern Europe, Princeton, Princeton University Press, 1994, p. 4.

3. M. Berman et J. Johnson (dir.), Unofficial Diplomats, New York, Columbia University Press, 1977, p. 31.

4. B. Badie, «Le jeu triangulaire », in P. Birnbaum (dir.), Sociologie des nationalismes, Paris, PUF, 1997, p. 448.

5. J.-F. Bayart, Le gouvernement du monde. Une critique politique de la globalisation, Paris, Fayard, 2004, p. 30 sq.

6. M. Keck et K. Sikkink, « Historical Precursors to Modern Transnational Social Movements and Networks », in J. Guidry, M. Kennedy et M. Zald (dir.), Globalization and Social Movements. Culture, Power, and the Transnational Public Sphere, Ann Arbor, University of Michigan Press, 2000, p. 35-53.

7. A. Becker, "Diplomacy Without Sovereignty : the World Jewish Congress Rescue Activities", in S. I. Troen et B. Pinkus (dir.), National Jewish Solidarity in the Modern Period, Londres, Frank Cass, 1992, p. 343-360.

8. D. Newsom (dir.), Private Diplomacy with the Soviet Union, Lanham, University of America Press, 1987.

9. A. Brysk, From Tribal Village to Global Village. Indian Rights and International Relations in Latin America, Stanford, Stanford University Press, 2000.

10. L. Gayer, «Les politiques mondiales de l'identité. Significations internationales des mobilisations identitaires des sikhs (Inde) et des Mohajirs (Pakistan) », Thèse de science politique (relations internationales), IEP de Paris, juillet 2004, notamment la deuxième partie.

11. M. Berman et J. Johnson (dir.), op. cit.

12. F. Constantin, "La privatisation de la politique étrangère : à partir de la scène africaine ", Pouvoirs, n88, 1999, p. 45.

13. H. Bozarslan, La question kurde. États et minorités au Moyen-Orient, Paris, Presses de Sciences Po, 1997.

14. G. Devin, "Les diplomaties de la politique étrangère », in F. Charillon (dir.), Politique étrangère. Nouveaux regards, Paris, Presses de Sciences Po, 2002, p. 234. 
15. Au printemps 1999, quelques mois après l'accord indo-pakistanais signé en février à Lahore entre les Premiers ministres des deux pays, l'Inde découvre que des combattants pakistanais ont pris position pendant l'hiver sur les sommets qui dominent la ville de Kargil, du côté indien de la Ligne de contrôle qui coupe le Cachemire en deux. Le Pakistan parle alors d'actions de mujahidin. S'ensuit une guerre limitée entre Inde et Pakistan, qui s'achève en juillet. Dans l'autobiographie qu'il vient de publier, le général Musharraf reconnaît que l'armée régulière pakistanaise s'est trouvée directement impliquée dans les opérations de Kargil. Selon le général-président, elle aurait alors déployé 5 bataillons ( 5000 hommes). Les autorités militaires indiennes ont, aussitôt le livre paru, démenti ces chiffres, affirmant qu'au moins 7 bataillons pakistanais auraient alors été mobilisés; voir S. Rajagopalan, «Proud Creator of Kargil », Hindustan Times (New Delhi), 26 septembre 2006, p. 1 .

16. G. Devin, art. cit., p. 216.

17. Par "mobilisation politique", ou "mouvement social", nous entendons ici l'action collective d'un groupe contestataire, orientée vers le changement social; voir L. Mathieu, Comment lutter? Sociologie et mouvements sociaux, Paris, Textuel, 2004, p. 17-25.

18. F. Constantin, "Affaires de familles et affaires d'État: à propos des modes populaires d'action diplomatique en Afrique orientale ", Revue française de science politique, vol. $36, \mathrm{n}^{\circ} 4$, 1986, p. 672-694.

19. J.-F. Bayart, C. Toulabor et A. Mbembe, Le politique par le bas en Afrique noire. Contribution à une problématique de la démocratie, Paris, Karthala, 1992.

20. Ces mobilisations de "victimes ", avec toute l'ambiguïté qui entoure ce statut convoité sur la scène internationale contemporaine, font actuellement l'objet d'une attention sans précédent de la science politique, comme l'a montré le colloque «Mobilisations de victimes ", qui s'est tenu à Paris X et Paris I les 15 et 16 juin 2006 sous la coordination scientifique de S. Lefranc et L. Mathieu, et dont les actes devraient être publiés en 2007.

21. F. Constantin, «L'informel internationalisé ou la subversion de la territorialité », Cultures \& Conflits, n 21-22, printemps-été 1996, p. 311-345.

22. G. Devin, art. cit., p. 236.

23. B. Hibou, «Retrait ou redéploiement de l'État?", Critique internationale, n 1, automne 1998, p. 163.

24. La notion de "société politique » fait référence aux institutions qui " agrègent les intérêts sociaux des groupes sociaux dans la sphère publique » et inclut donc les partis politiques, mais aussi, comme le propose C. Jaffrelot, les syndicats, les associations et les médias, voire l'appareil judiciaire ; voir C. Jaffrelot, "L'Inde et le Pakistan : l'impossible convergence démocratique de deux trajectoires politiques", in J. Santiso (dir.), À la recherche de la démocratie. Mélanges offerts à Guy Hermet, Paris, Karthala, 2002, p. 179. Sous une acception plus restrictive, focalisée sur les partis politiques, la notion est apparue dans J. Linz et A. Stepan, Problems of Democratic Transition and Consolidation. Southern Europe, South America and Post-Communist Europe, Baltimore, The Johns Hopkins University Press, 1996, p. 8.

25. Le plus célèbre de ces réseaux de pacificateurs diasporiques est le South Asia Citizens Web, animé de France par Harsh Kapoor. On pourra consulter le site de ce forum à l'adresse suivante : http://www.sacw.net/.

26. On trouvera une sélection d'articles et de communiqués des pacifistes indiens et pakistanais dans S. Kothari et Z. Mian (dir.), Out of the Nuclear Shadow, Delhi et Londres, Lokayan, Rainbow Publishers et Zed Books, 2001.

27. Une enquête publiée par le magazine India Today au lendemain des essais nucléaires de 1998 soulignait que $47 \%$ des Indiens ignoraient tout de ces tests; voir "Making of a Wave ", India Today (New Delhi), 28 décembre 1998. 
28. S. Roy, «Nuclear Frames: Official Nationalism, the Nuclear Bomb, and the Anti-Nuclear Movement in India », in M. V. Ramana, et C. Rammanohar Reddy (dir.), Prisoners of the Nuclear Dream, Delhi, Orient Longman, 2003, p. 333-359.

29. A. Becker, art. cit.

30. L. Gayer, op. cit.

31. Les Mohajirs sont les descendants des migrants musulmans indiens qui se sont établis dans les grands centres urbains du Sind (Karachi et Hyderabad, notamment) après la Partition. Cette population ourdouphone a d'abord partagé le pouvoir avec les Pendjabis, avant de connaitre une période de relatif déclin politique et économique à la suite de l'émergence des Sindis sur la scène politique pakistanaise.

32. Le MQM, fondé en 1984, est issu d'un syndicat étudiant, la All Pakistan Mohajir Students Organisation (APMSO), fondée en 1978 par des étudiants en médecine et en pharmacie de Karachi.

33. En arabe, le terme murid désigne littéralement « celui qui désire ». Dans la tradition soufie, le murid est celui qui se soumet à l'autorité de son chef spirituel (pir, murshid, shaikh...), après avoir été admis dans la confrérie au terme d'un parcours initiatique ; voir John L. Esposito (dir.), The Oxford Dictionary of Islam, New York, Oxford University Press, 2003, p. 215-216.

34. D. Cefaï, «Expérience, culture et politique », in D. Cefaï (dir.), Cultures politiques, Paris, PUF, 2001, p. 102-103.

35. L. Gayer, « Un turban pour les Mounties : multiculturalisme canadien et politiques du corps sikhes », Raisons politiques ( $1^{\text {re }}$ formule), $n^{\circ} 4,2000$, p. 67-90.

36. Pour une analyse plus approfondie des diplomaties identitaires des sikhs et des Mohajirs, nous renvoyons à L. Gayer, « Projections internationales ou détours vers le local ? Les diplomaties identitaires des sikhs (Inde) et des Mohajirs (Pakistan)», International Review of Sociology, à paraître fin 2007.

37. Le maulana Fazl-ur Rehman, leader de l'opposition pakistanaise depuis les élections de 2002, s'est ainsi rendu en Inde en 2003 et en 2006 avec une mission diplomatique officieuse, consistant à explorer des pistes de négociation avec la partie indienne.

38. Sur le sport comme diplomatie parallèle, nous renvoyons à J.-F. Polo, « "Avrupa Fatih" (Les conquérants de l'Europe): les enjeux européens du sport en Turquie", in L. Arnaud et C. Guionnet (dir.), Les frontières du politique. Enquête sur les processus de politisation et de dépolitisation, Rennes, Presses universitaires de Rennes, 2005, p. 209-232.

39. Cité dans R. Bhattacharya, Pundits from Pakistan. On Tour with India, 2003-2004, Londres, Pan Macmillan, 2005, p. 11.

40. En 2004, de nombreux chauffeurs de taxis, commerçants et hôteliers pakistanais ont refusé de facturer leurs services aux visiteurs indiens. En retour, de nombreux Pendjabis sikhs ont offert le gîte aux visiteurs pakistanais venus assister au match de l'équipe indienne et de sa rivale pakistanaise à Mohali, au cours du printemps 2005.

41. Pour un compte rendu de cette rencontre, voir S. Khan, Cricket, a Bridge of Peace, Karachi, Oxford University Press, 2005.

42. La Nouvelle-Zélande a été contrainte d'annuler la tournée de son équipe nationale en mai 2002, après un attentat (contre un bus de la Direction des constructions navales française) à proximité de l'hôtel où celle-ci se trouvait logée à Karachi.

43. SFGate.com, 4 mars 2004.

44. Vigilonline.com, 22 décembre 2003.

45. «Cricket as holy grail», Hard News (New Delhi), février 2006, p. 40.

46. Dans un contexte bilatéral marqué par un regain de tensions, à la suite des déclarations du gouvernement indien enjoignant aux autorités pakistanaises de faire preuve de retenue dans le traitement du dossier baloutche, le Premier ministre indien, Manmohan Singh, a décliné 
l'invitation du général Musharraf à se rendre au Pakistan pour assister à l'un des matchs entre les deux équipes.

47. Sur ce point, nous renvoyons à A. Blom, «Pakistan's Islamist Warriors : a Comparative Perspective ", in C. Jaffrelot et S. V. R. Nasr (dir.), Balancing Stability and Change in Pakistan. Political Reform, Islamic Activism and Development Scenarios, en préparation.

48. S. N. Prasad et D. Pal, History of Operations in Jammu \& Kashmir (1947-48), History Division, Ministry of Defence, Government of India et Natraj Publishers, 1987.

49. A. Khan, Raiders in Kashmir. Story of the Kashmir War (1947-48), Karachi, Pak Publishers, 1970.

50. Au cours d'une émission de la BBC diffusée en 1997, l'un des collaborateurs de Jinnah, Shaukat Ayat Khan, alors ministre dans le gouvernement du Pendjab, confirma que Jinnah avait bien donné l'ordre d'envahir le Cachemire; voir C. Dasgupta, War and Diplomacy in Kashmir, 1947-48, New Delhi, Sage, 2002, p. 219, note 10.

51. Dessinée en 1893 pour séparer l'empire des Indes de l'Afghanistan, la ligne Durand correspond aujourd'hui à la frontière entre le Pakistan et l'Afghanistan.

52. A. S. Ahmed, "Pukhtun Tribes in the Great Game", in Pakistan Society. Islam, Ethnicity and Leadership in South Asia, Delhi, Oxford University Press, 1988, p. 133-157.

53. R. Hussain, Pakistan and the Emergence of Islamic Militancy in Afghanistan, Aldershot, Ashgate, 2005, p. 39-40.

54. I. Talbot, Pakistan. A Modern History, Londres, Hurst, 1998, p. 94, 13.

55. S. V. R. Nasr, International Relations of an Islamist Movement. The Case of Jamaat-i-Islami of Pakistan, New York, Council on Foreign Relations, 2000, p. 32-33.

56. En 1973, le général Babar avait été chargé d'entraîner les premiers rebelles islamistes afghans dans des camps mis à la disposition des insurgés par l'armée pakistanaise ; R. Hussain, op. cit., p. 79.

57. Sur cette autonomisation progressive des talibans vis-à-vis de leur patron pakistanais, nous renvoyons à G. Dorronsoro, «La grande illusion : bilan de la politique afghane du Pakistan », Les Études du CERI, $\mathrm{n}^{\circ} 84$, mars 2002.

58. L. Gayer, "Guns, Slums and "Yellow Devils" : a Genealogy of Urban Conflicts in Karachi, Pakistan », Modern Asian Studies, à paraître début 2007.

59. H. Haqqani, Pakistan. Between Mosque and Military, Washington, Carnegie Endowment for International Peace, 2005, p. 267.

60. R. Hussain, op. cit., p. 122.

61. H. Haqqani, op. cit., p. 273.

62. Comme le relève Will $\mathrm{H}$. Moore, " un groupe doit d'abord se battre seul avant que d'autres pays acceptent de lui venir en aide sous une autre forme que celle d'un simple soutien symbolique ou de pressions diplomatiques »; voir W. H. Moore, «Ethnic Minorities and Foreign Policy ", SAIS Review, 22 (2), été-automne 2002, p. 82.

63. En juin 1984, l'armée indienne prit d'assaut le Temple d'or d'Amritsar, haut lieu du sikhisme, où s'étaient retranchés les leaders du mouvement sécessionniste du Khalistan.

64. Entretien, Ludhiana, 19 mars 2001.

65. Entretien, Patiala, 29 mars 2001.

66. La relation du commandant de la KCF avec l'ISI fut parfois houleuse et il quitta le Pakistan en 1992 après avoir refusé de retourner au Pendjab indien pour y prendre la tête de la lutte armée ; voir H. S. Bhanwer, « Militants being trained by China », The Tribune (Chandigarh), 9 décembre 1992.

67. Entretien, Londres, 22 juillet 1999.

68. L'ISI encouragea même les militants du Hizb-ul Mujahidin à dénoncer voire à éliminer les cadres du JKLF ; voir H. Haqqani, op. cit., p. 290.

69. Ibid., p. 288-289. 
70. Dans le contexte pakistanais, le sectarisme se réfère au conflit entre les groupes armés sunnites et chiites apparus au cours des années 1980. Ces organisations sont principalement implantées au Pendjab et dans le Sind; voir M. Q. Zaman, "Sectarianism in Pakistan: the Radicalization of Shii and Sunni Identities », Modern Asian Studies, 32(3), 1998, p. 689-716.

71. "Yet to see India's passion for peace », entretien avec Shaukat Aziz, Outlook (New Delhi), 7 août 2006, p. 44.

72. B. Hocking, «Catalytic Diplomacy: Beyond "Newness" and "Decline" », in J. Melissen (dir.), Innovation in Diplomatic Practice, Houndmills, Palgrave, 1999, p. 21-42.

73. L'apolitisme revendiqué des sportifs professionnels constitue une forme de positionnement politique et peut les conduire à résister aux tentatives d'instrumentalisation des États. Moins que leur prétendu "apolitisme », c'est donc surtout le pacifisme affiché par ces acteurs qui facilite leur recrutement par l'État, lorsque ses objectifs pacificateurs convergent avec l'ethos sportif. Sur ce rapport des sportifs au politique, voir J. Defrance, «La politique de l'apolitisme: sur l'autonomisation du champ sportif », Politix, vol. 13, n50, 2000, p. 14-27.

\section{INDEX}

Index géographique : Inde, Pakistan

Mots-clés : politique étrangère, diplomatie parallèle, acteurs non-étatiques

Keywords : India, Pakistan, foreign policy, parallel diplomacy, non-state actors 